\title{
Pooling knowledge to tailor an integrated delivery system
}

\author{
Linda D Bosserman, MD, FACP
}

W hat an exciting and challenging year 2014 has been! As it draws to a close, we also celebrate the first year of the merger between The Journal of Supportive Oncology and Community Oncology to form our current title, The Journal of Community and Supportive Oncology. We hope that by combining the clinical and supportive/palliative components of our specialty, we are able to serve as a vital forum and resource by providing you with a "one-stop shop" to support you in your practice of oncology.

\section{The practice front}

We have seen the continued consolidation of oncology practices across the country this year as people pilot different models of reimbursement and more integrated delivery systems. Although the goals of all clinicians remain to provide high value, compassionate, personalized care, oncology has been especially hard pressed to do this more cost effectively.

A major challenge remains the lack of data integration across offices, hospitals, labs, radiology, and health plan systems. Practices have been forced to enter data into multiple payer web sites for authorizations and to use different pathway systems, which has shifted a greater administrative burden to medical oncologists. Physicians are being asked to enter every order specifically rather than ordering standardized regimen and using care plans with details populated by the electronic medical record (EMR) and confirmed by licensed nurses or advanced practice providers. EMRs are still used more for data entry, therapy regimens, prescriptions, and proving specific compliance with Medicare measures than as tools to enhance knowledge at the point of care. Those tools would assist in sorting the increasing number of therapies for more targeted patient and tumor characteristics, providing more comprehensive and continuous symptom management, understanding real-life outcomes in sets of patients with various medications and comorbidities who receive cancer therapies, as well as better survival and end-of-life care.

The electronic health record (EHR) has the potential to better support caregivers, researchers, payers, and admin- istrators, but we need to agree to standardized data sets. Comprehensive data sets can help us evaluate value: what populations we serve; what their diagnoses, comorbidities, medications, and therapies are; and what the outcomes and costs are. As the American Society of Clinical Oncology launches CancerLinQ in early 2015 to aggregate oncology data from across the United States, and as EMRs expand analytic reports from entered data and IT specialty groups aggregate oncology data from multiple practices, we can expect to have a more specific understanding of the clinical and financial outcomes for our patients.

Another immediate challenge in this area is how to pool the knowledge and best practices among the users of the numerous EMRs and EHRs. For example, many hospitals or clinics that are using systems such as Epic and Sunrise have been advised by their legal teams that they cannot collaborate and share programming. This is against one of our most cherished principles, that of being able to freely share medical information that helps us better and more efficiently use our limited resources to improve patient care. Given that the NCCN (National Comprehensive Cancer Network) guidelines are a compendium of evidence-based guidelines for the country and that the regimens specified in them are drawn from clinical research papers that specify drugs, doses, schedule, and cycle numbers, it would be a massive time saving to be able to share the building process of regimens and order sets across systems using similar EMRs or EHRs. Getting groups that are on common platforms to share best practices is how many community practices that share common oncology-specific EMRs have been able to more rapidly implement the systems and begin to draw on informative data.

I believe that the clinician community needs to speak up, get together, and begin helping each other devise and oversee the use of EMRs to improve patient outcomes and safety as well as to streamline efficiencies in data entry so that clinicians can focus on their unique skills and training to evaluate data that will inform them in their development of personalized patient care plans. It will take legisla- 
tive action or new payment models to facilitate and pay for team-based care and efficiencies to achieve quality, comprehensive, cost-effective care with better outcomes and full patient engagement.

\section{The therapeutic armamentarium}

While the oncology care delivery system grapples with the aforementioned challenges, the 2014 therapeutic landscape was enriched with the US Food and Drug Administration's approval of 9 new drugs and of expanded uses or formulations for another 3 drugs.

\section{New approvals}

- Pembrolizumab (Keytruda) was granted accelerated approval for treating patients with unresectable or metastatic melanoma with disease progression after treatment with ipilumumab or - for patients who are BRAF V600 mutated - after treatment with a BRAF inhibitor.

- Trametinib (Mekinist) and dabrafenib (Tafinlar) were approved for use in combination for patients with unresectable or metastatic melanoma with a BRAF V600E or $\mathrm{V} 600 \mathrm{~K}$ mutation as detected by an FDA-approved test.

- Idelalisib (Zydelig) was approved for use patients with relapsed chronic lymphocytic leukemia (CLL) in combination with rituximab.

- Ofatumumab (Arzerra) was approved in combination with chlorambucil for treatment of previously untreated patients with CLL for whom fludarabine-based therapy is considered inappropriate.

- Belinostat (Beleodaq) was granted accelerated approval for patients with relapsed or refractory peripheral T-Cell lymphoma.

- Ceritinib (Zykadia) was granted accelerated approval for anaplastic lymphoma kinase and positive metastatic non-small-cell lung cancer with progression or intolerance on crizotinib (Xalkori).

- Siltuximab (Sylvant) was approved for treatment of patients with multicentric Castelmen's disease who are negative for HIV and human herpes virus 8.

- Ramucirumab (Cyramza) was approved for use in patients with advanced or metastatic gastric or gastroesophageal junction adenocarcinoma who progressed on or after treatment with fluropyrimidine or platinumcontaining chemotherapy.

\section{Additional indications}

- Ibrutinib (Imbruvica) received accelerated approval for patients with CLL who have received at least one prior therapy - an expansion of its previous approval for patients with mantle cell lymphoma who had at least one prior therapy.

- An oral suspension of mercaptopurine (Purixan) was approved for patients with acute lymphoblastic leukemia as part of a combination regimen.

- Bevacizumab (Avastin) received expanded approval for the treatment of persistent, recurrent, or metastatic cervical cancer in combination with paclitaxel and cisplatin or paclitaxel and topotecan with a significant survival advantage accompanied by significant toxicities.

We close this year with our best wishes to you and your families, staff, and patients for a holiday season celebrating another year of delivering quality, compassionate care in the midst of numerous clinical and administrative challenges and policy changes. We are able to draw on a growing armamentarium of increasingly targeted therapies and look forward to the promise of significantly more effective and less toxic therapies in the pipeline. In the coming year, we should challenge each other to pool our knowledge so that we can pioneer a more effective and efficient team-based delivery system. We need to get highly trained clinicians back to their primary focus: their patients. For that to happen, providers need to have integrated clinical teams and the kind of administrative and payer support to curate the evidence-based data needed to grow and support a more patient-centered delivery system. From us to you, happy 2015 ! 